\title{
Numerical Optimization of a Cylindrical Reflector-in-Radome Antenna System
}

\author{
Vladimir B. Yurchenko, Ayhan Altintaş, Senior Member, IEEE, and Alexander I. Nosich, Senior Member, IEEE
}

\begin{abstract}
Accurate numerical optimization based on the rigorous solution of the integral equation using the method of analytical regularization is performed for the cylindrical reflector antenna in a dielectric radome. It is shown that the multiple scattering in this system is more significant for the optimum radome design than any nonplane-wave effects or the curvature of the radome. We claim that, although the common half-wavelength design is a good approximation to avoid negative effects of the radome (such as the loss of the antenna directivity), one can, by carefully playing with the radome thickness, its radius, reflector location, and the position of the feed, improve the reflector-inradome antenna performance (e.g., increase the directivity) with respect to the same reflector in free-space.
\end{abstract}

Index Terms-Moment methods, radomes, reflector antennas.

\section{INTRODUCTION}

$\mathbf{T}$ $O$ ensure protection from dust, wind, rain, and snow, reflector antennas are commonly covered with spherical dielectric radomes [1]. Design of such systems is a complicated engineering task, while an accurate theoretical analysis and optimization is a really challenging problem. Normally a number of assumptions is introduced in order to make it treatable. One of the earliest ideas was an approximate description of the radome by using the resistive boundary condition [2] derived for a very thin dielectric shell, and solving the problem by the method of moments (MoM). The other traditional techniques for the analysis of radomes are the plane-wave spectrum, surface integration [3], and the ray tracing [4]-[6]. Both are high-frequency asymptotic techniques treating the aperture field distribution as a series of rays that are traced through the radome wall. In [5], [6], ray tracing was extended to include the curvature effects of a circular cylindrical radome. Instead of locally flat, a locally circular-cylindrical surface model was proposed in [7]. In [8], the finite-element technique was used to predict the effect of radome on the far-field pattern. Within any of these approaches, the multiple scattering between the source, reflector, and radome is ignored. If any optimization is performed, it is made for each element of the system separately.

In general, it is considered that the presence of the radome in the near zone of reflector is a negative factor in electromag-

Manuscript received October 2, 1997; revised October 29, 1998.

V. B. Yurchenko is with the Institute of Radiophysics and Electronics, National Academy of Sciences of Ukraine, Kharkov 310085, Ukraine, and with the Department of Electrical and Electronics Engineering, Bilkent University, Bilkent, Ankara 06533, Turkey.

A. Altintaş is with the Department of Electrical and Electronics Engineering, Bilkent University, Bilkent, Ankara 06533, Turkey.

A. I. Nosich is with the Institute of Radiophysics and Electronics, National Academy of Sciences of Ukraine, Kharkov 310085, Ukraine.

Publisher Item Identifier S 0018-926X(99)04773-0. netic sense. Antenna performance normally suffers: the main beam can be distorted, sidelobe level gets higher, and overall parameters such as the directivity are lost. A well-known engineering "rule-of-thumb" tells that the radome thickness should be taken as $1 / 2$ of the wavelength in the radome material, to minimize the distortions. The radome curvature may effect this rule, especially for smaller radomes. Free-space analysis tells that the feed should be shifted toward the reflector [9] due to the spillover and aberrations. There is no simple rule, unfortunately, to predict the effect of reflector and feed positioning inside the radome. Approximate high-frequency techniques are very cumbersome if several scatterers are involved.

Thus, in fact, such antenna systems are not designed in a self-consistent manner, by taking fully into account all the interactions between the elements. In this paper, we analyze a two-dimensional (2-D) problem of this kind by means of a rigorous integral equation approach applied to the case of H-polarization mode. The method of solution is based on the combination of the complex source-point (CSP) technique [10], Green's function technique, and the method of regularization (MoR), and is a further development of our previous work [11]. The case of E-polarization can be considered in a similar way. The difference will be in using the single-layer potential representation for the radiated field instead of the double-layer one [11].

We demonstrate that an accurate numerical optimization procedure can bring a different vision of the radome effect. By a clever play with the radome thickness, its radius, reflector location inside the radome, and the position of the feed, one can improve antenna performance with respect to the freespace reflector. To reach this goal, the optimization code must be based on solving the integral equation by means of the numerically exact technique such as MoR providing a guaranteed accuracy of the solution.

By implementing the MoR technique, we show, in particular, that the directivity of the antenna in the radome can be increased as compared to the same antenna in free-space due to the effect of multiple scattering arising in the entire reflector-in-radome system.

Within the same approach, one can rigorously analyze the effect of the radome on other characteristics of antenna, e.g., on the VSWR at the feed determined by the partial wave reflection from the radome [12], [13]. However, we do not study these effects in the present paper since our focus is on the radiation characteristics.

Although cylindrical reflector antennas are in less wide use than spherical and paraboloidal ones, they find applications in 


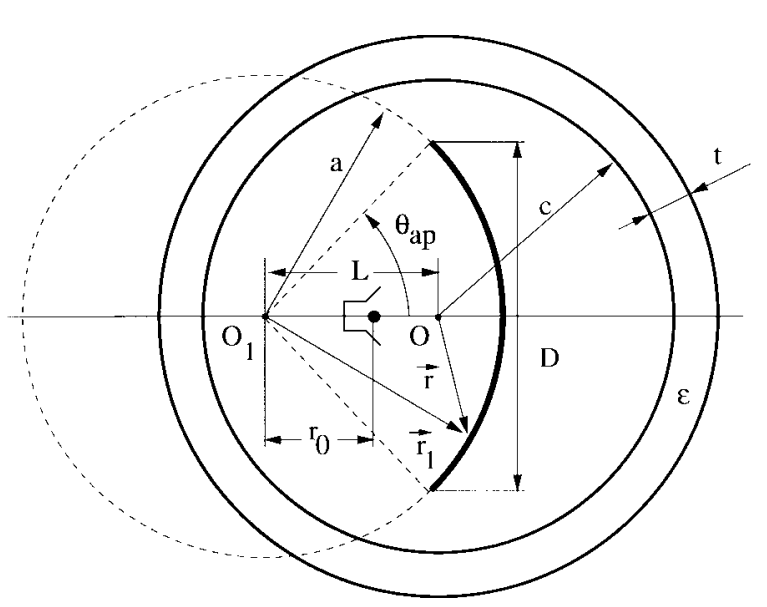

Fig. 1. Geometry of the 2-D reflector in a circular dielectric radome $\left(D=2 a \sin \left(\theta_{a p}\right), f=0.5 a\right.$ is the focal distance).

airborne navigational antenna design where sharp azimuthal beams and wide-angle vertical coverages are required [14].

Three-dimesional spherical-reflector antenna in a spherical radome can also be optimized along similar lines. However, in this case, analytical regularization should be based on a different technique [15], and on the use of addition theorems for spherical wave functions.

The remainder of this paper is organized as follows. In Section II we formulate the problem of $\mathrm{H}$-polarized radiation in terms of the full-wave electric-field integral equation (IE). The IE kernel is determined by the Green's function of the circular dielectric radome. We obtain this function in explicit form using the addition theorems for the cylindrical functions. Then, assuming that the reflector contour is a circular arc, we cast the IE to the series equations and invert the static part of the latter. This yields a regularized matrix equation for the surface current expansion coefficients. In Section III, we first present some numerical results for the far-field radiation pattern of reflector-in-radome antenna, and plot the directivity as a function of geometrical parameters. Further, we use this algorithm for a computer-aided optimization of the geometry, having the maximum directivity as an aim function. Section IV contains some conclusions of the reported work. A note should be made that the time dependence is assumed as $e^{-i \omega t}$ and omitted throughout the paper.

\section{Problem Formulation And Solution}

Consider a 2-D model of antenna shown in Fig. 1, with cylindrical reflector arbitrary located inside a circular cylindrical dielectric radome. Relative dielectric permittivity of the radome is $\epsilon_{r}$ (relative permeability $\mu_{r}=1$ ), inner radius $c$, and outer radius $d$. The feed is assumed to be a complex-point line source radiating a directive incident beam. The beamwidth is determined by the imaginary part of the source coordinate, which simulates the aperture width of a realistic horn feed (see [10]). The feed is assumed to be located on the axis of symmetry of reflector-in-radome antenna. Hence the free-space field of such a CSP feed is given by

$$
H^{i n}(\vec{r})=H_{0}^{(1)}\left(k\left|\vec{r}-\vec{r}_{s}\right|\right)
$$

where $k$ is the free-space wavenumber, $\vec{r}_{s}=\vec{r}_{0}+i \vec{b}$ is the complex source position, $\vec{r}_{0}$ is the real-space source position, and $\vec{b}$ is the beam parameter vector. In the polar coordinates $\vec{r}_{s}=\left(r_{s}, \varphi_{s}\right), \vec{r}_{0}=\left(r_{0}, \varphi_{0}\right)$, and $\vec{b}=(b, \beta)$ where $b$ and $\beta$ determine the beam width and orientation, respectively. For a symmetric feeding, $\varphi_{0}=0$ or $\pi$, and $\beta=0$. Assuming that $\operatorname{Re}\left(r_{s}\right)>0$ and $b>0$ one finds that

$$
\begin{aligned}
r_{s} & =\left[r_{0}^{2}+i 2 r_{0} b \cos \left(\beta-\varphi_{0}\right)-b^{2}\right]^{1 / 2} \\
\varphi_{s} & =\arccos \left[\left(r_{0} \cos \varphi_{0}+i b \cos \beta\right) / r_{s}\right] .
\end{aligned}
$$

The directivity and the total radiated power of such a source are $D_{0}=I_{0}^{-1}(2 k b) e^{2 k b}$ and $P_{0}=2 Z_{0} k^{-1} I_{0}(2 k b)$ [11], respectively, where $I_{0}(\cdot)$ is the modified Bessel function and $Z_{0}$ is the intrinsic impedance.

In the presence of a reflector enclosed in the radome, the CSP field is to be completed by the scattered field $H^{s c}$ yielding the total field

$$
H^{\text {tot }}=H^{i n}+H^{s c} .
$$

The requirements for (3) to be a solution of the considered problem are stated as satisfying the 2-D Helmholtz equation, the Sommerfeld radiation condition at $r \rightarrow \infty$, the Meixner condition at the reflector edges, the Neumann boundary condition at the reflector surface $M$, and the continuity conditions for the functions $H^{\text {tot }}$ and $\epsilon_{r}^{-1} \partial H^{\text {tot }} / \partial n$ at the inner and outer surfaces of the radome.

To meet the requirements, we seek the function $H^{s c}$ in the form

$$
H^{s c}\left(\vec{r}_{1}\right)=\int_{M} j\left(\vec{r}_{1}^{\prime}\right) \frac{\partial}{\partial n^{\prime}} G\left(\vec{r}_{1}, \vec{r}_{1}^{\prime}\right) d r_{1}^{\prime}
$$

where $G$ is the Green's function of the circular dielectric shell. The function $G$ takes rigorously into account the geometry and material parameters of the radome including the curvature effect, creeping waves, resonances, and the radiation condition at $r \rightarrow \infty$.

In the case of a circular radome, the Green's function can be obtained analytically. Indeed, it is found as a solution to the problem of excitation of the radome by a magnetic line source. In the radome coordinates $(r, \varphi)$, the excited magnetic field $H^{\operatorname{Rad}}(\vec{r})$ is expanded as

$$
H^{\operatorname{Rad}}(\vec{r})=\sum_{n=-\infty}^{\infty}\left\{\begin{array}{c}
A_{n} J_{n}(k r) \\
B_{n} J_{n}\left(k_{\epsilon} r\right)+C_{n} H_{n}^{(1)}\left(k_{\epsilon} r\right) \\
D_{n} H_{n}^{(1)}(k r)
\end{array}\right\} \epsilon^{i n \varphi}
$$

where each of the lines refers to the relevant region of $r<c, c \leq r \leq d$ or $r>d, J_{n}(\cdot)$ and $H_{n}^{(1)}(\cdot)$ are the Bessel and Hankel functions, respectively, and $k_{\epsilon}=\sqrt{\epsilon_{r}} k\left(\lambda_{\epsilon}=\lambda / \sqrt{\epsilon_{r}}\right.$ is the wavelength in the radome material). In the reflector coordinates $\left(r_{1}, \varphi_{1}\right)$

$$
\begin{aligned}
& H^{\operatorname{Rad}}\left(\vec{r}_{1}\right)=\sum_{n, p=-\infty}^{\infty} \\
& \cdot\left\{\begin{array}{c}
A_{n} J_{p}\left(k r_{1}\right) \\
B_{n} J_{p}\left(k_{\epsilon} r_{1}\right)+C_{n} H_{p}^{(1)}\left(k_{\epsilon} r_{1}\right) \\
D_{n} H_{p}^{(1)}\left(k r_{1}\right)
\end{array}\right\} \\
& \text { - } J_{p-n}(k L) e^{i p \varphi_{1}}
\end{aligned}
$$


where $L$ is the shift of the radome with respect to reflector $\vec{r}_{1}=\vec{r}+\vec{L}$ (Fig. 1). When the boundary conditions at the radome surfaces are satisfied and the expansion coefficients found, (5) and (6) represent the Green's function being of interest.

The electric-field IE is obtained from (3) by applying the PEC boundary condition on the contour $M$ of the reflector (H-polarization)

$$
\frac{Z_{0}}{i k} \frac{\partial}{\partial n} \int_{M} j\left(\vec{r}_{1}^{\prime}\right) \frac{\partial}{\partial n^{\prime}} G\left(\vec{r}_{1}, \vec{r}_{1}^{\prime}\right) d \vec{r}_{1}^{\prime}=-E_{t g}^{i n}\left(\vec{r}_{1}\right)
$$

where $Z_{0}$ is the free-space impedance, $j\left(\vec{r}_{1}^{\prime}\right)$ is the current density function to be found, $E_{t g}^{i n}=\left(Z_{0} / i k\right) \partial H^{i n} / \partial n$ is the tangential component of the CSP electric field at the reflector surface, and $\vec{n}$ is the outer unit normal vector.

Note that in IE (7) we need only the Green's function with both the source location point $\vec{r}_{1}^{\prime}$ and the observation point $\vec{r}_{1}$ inside the radome $\left(r^{\prime}, r<c\right)$. Expanding this function in terms of the azimuthal harmonics in the radome coordinates $(r, \varphi)$ and satisfying the continuity conditions on the radome surfaces, we obtain

$$
G\left(\vec{r}, \vec{r}^{\prime}\right)=G^{0}\left(\vec{r}, \vec{r}^{\prime}\right)+G^{H}\left(\vec{r}, \vec{r}^{\prime}\right)
$$

where $G^{0}=(i / 4) H_{0}^{(1)}\left(k\left|\vec{r}-\vec{r}^{\prime}\right|\right)$ is the 2-D free-space Green's function and $G^{H}$ is the term accounting for the presence of the radome in the $H$-polarization mode. When transformed back to the reflector coordinate system, the Green's function $G\left(\vec{r}, \vec{r}^{\prime}\right)$ takes the form

$$
\begin{aligned}
G\left(\vec{r}_{1}, \vec{r}_{1}^{\prime}\right)= & \frac{i}{4} H_{0}^{(1)}\left(k\left|\vec{r}_{1}-\vec{r}_{1}^{\prime}\right|\right) \\
& -\frac{i}{4} \sum_{n=-\infty}^{\infty} J_{n}\left(k r_{1}\right) e^{i n \varphi_{1}} \\
& \cdot \sum_{m=-\infty}^{\infty} L_{m n} J_{m}\left(k r_{1}^{\prime}\right) e^{-i m \varphi_{1}^{\prime}}
\end{aligned}
$$

where the condition $L<\min (a, c)$ is assumed in order to present $G\left(\vec{r}_{1}, \vec{r}_{1}^{\prime}\right)$ as a single formula everywhere inside the radome. The factor $L_{m n}$ in (9) accounts for the shift of the reflector from the concentric position

$$
L_{m n}=\sum_{p=-\infty}^{\infty} K_{p} J_{m-p}(k L) J_{n-p}(k L)
$$

and the coefficients $K_{p}$ account for the presence of the radome in $H$-polarization mode

$$
K_{p}=\left(g_{p} c_{p}-f_{p} d_{p}\right) / Q_{p}
$$

where $Q_{p}=g_{p} a_{p}-f_{p} b_{p}$

$$
\begin{aligned}
f_{p} & =J_{p}\left(k_{\epsilon} d\right) H_{p}^{\prime}(k d)-\eta J_{p}^{\prime}\left(k_{\epsilon} d\right) H_{p}(k d) \\
g_{p} & =H_{p}\left(k_{\epsilon} d\right) H_{p}^{\prime}(k d)-\eta H_{p}^{\prime}\left(k_{\epsilon} d\right) H_{p}(k d) \\
a_{p} & =J_{p}\left(k_{\epsilon} c\right) J_{p}^{\prime}(k c)-\eta J_{p}^{\prime}\left(k_{\epsilon} c\right) J_{p}(k c) \\
b_{p} & =H_{p}\left(k_{\epsilon} c\right) J_{p}^{\prime}(k c)-\eta H_{p}^{\prime}\left(k_{\epsilon} c\right) J_{p}(k c) \\
c_{p} & =J_{p}\left(k_{\epsilon} c\right) H_{p}^{\prime}(k c)-\eta J_{p}^{\prime}\left(k_{\epsilon} c\right) H_{p}(k c) \\
d_{p} & =H_{p}\left(k_{\epsilon} c\right) H_{p}^{\prime}(k c)-\eta H_{p}^{\prime}\left(k_{\epsilon} c\right) H_{p}(k c)
\end{aligned}
$$

$\eta=\sqrt{\mu_{r} / \epsilon_{r}}$, and $H_{p}(\cdot)=H_{p}^{(1)}(\cdot)$.

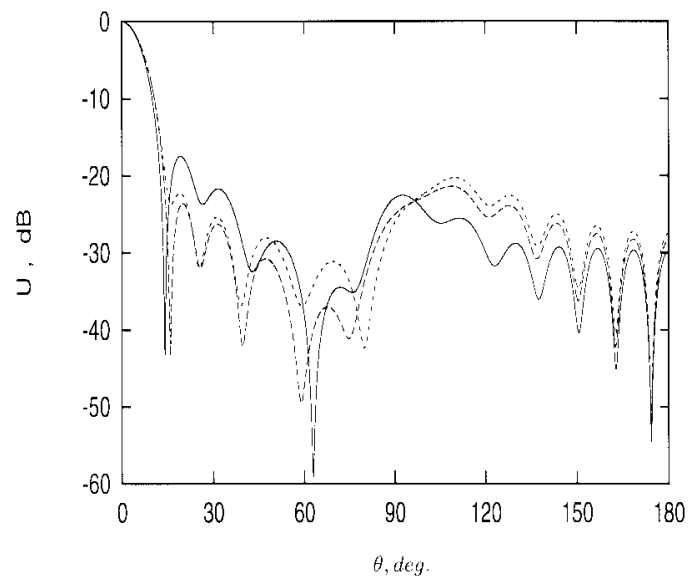

Fig. 2. Far-field patterns of the reflector in free space $\left(r_{0}=0.5 a\right.$, dotted curve, and $r_{0}=0.533 a$, dashed curve), and of the optimum reflector-in-radome antenna system $\left(r_{0}=0.560 a, t=0.341 \lambda_{\epsilon}\right.$, $L=4.271 \lambda, c=5.984 \lambda$, solid curve).

Further, we follow [11] and solve (7) by MoR. To avoid the numerical integrations for filling the resulting equation matrix, we take the geometry of parabolic reflector in such a way as to simulate it by a circular one of the radius $a$ and the angular width $2 \theta_{a p}$. The approximation is known to be acceptable if the geometries are chosen such that the electrical error does not exceed 1/16th of the wavelength [1] (see [11, Fig. 2] for the acceptable range of $k a$ and $\theta_{a p}$ parameters). This enables us to convert (7) to a discrete form through the so-called dual-series equations (DSE) in terms of the surface current expansion coefficients $x_{n}$ as in [11]

$$
\begin{aligned}
& \sum_{n=-\infty}^{\infty}\left[x_{n} H_{n}^{(1)^{\prime}}(k a)-y_{n}\right] J_{n}^{\prime}(k a) e^{i n \varphi_{1}} \\
&=-\sum_{n=-\infty}^{\infty} h_{n} e^{i n \varphi_{1}}, \quad \varphi_{1} \in M \\
& \sum_{n=-\infty}^{\infty} x_{n} e^{i n \varphi_{1}}=0, \quad \varphi_{1} \in S
\end{aligned}
$$

where

$$
\begin{aligned}
y_{n}= & \sum_{m=-\infty}^{\infty} x_{m} J_{m}^{\prime}(k a) L_{m n} \\
h_{n}= & J_{n}\left(k r_{s 1}\right) H_{n}^{(1)^{\prime}}(k a) e^{-i n \varphi_{s}}-J_{n}^{\prime}(k a) \\
& \cdot \sum_{m=-\infty}^{\infty} L_{m n} J_{m}\left(k r_{s 1}\right) e^{-i m \varphi_{s}} .
\end{aligned}
$$

Regularization of these equations is based on the analytical inversion of the static part taking an exact account of the edge behavior. We refer to [11] for details and present the final matrix equation

$$
x_{m}-\sum_{n=-\infty}^{\infty} x_{n}\left(A_{m n}^{0}+A_{m n}^{\mathrm{Rad}}\right)=B_{m}^{0}+B_{m}^{\mathrm{Rad}}
$$

where $m=0, \pm 1, \pm 2, \cdots$

$$
A_{m n}^{0}=T_{m n}\left(\theta_{a p}\right)\left(|n|+i \pi(k a)^{2} J_{n}^{\prime}(k a) H_{n}^{(1)^{\prime}}(k a)\right)
$$




$$
\begin{aligned}
A_{m n}^{\mathrm{Rad}}= & -i \pi(k a)^{2} J_{n}^{\prime}(k a) \sum_{p=-\infty}^{\infty} T_{m p}\left(\theta_{a p}\right) L_{n p} J_{p}^{\prime}(k a) \\
B_{m}^{0}= & i \pi(k a)^{2} \sum_{n=-\infty}^{\infty} T_{m n}\left(\theta_{a p}\right) H_{n}^{(1)^{\prime}}(k a) J_{n}\left(k r_{s 1}\right) e^{-i n \varphi_{s 1}} \\
B_{m}^{\mathrm{Rad}}= & -i \pi(k a)^{2} \sum_{n=-\infty}^{\infty} T_{m n}\left(\theta_{a p}\right) J_{n}^{\prime}(k a) \\
& \cdot \sum_{p=-\infty}^{\infty} L_{n p} J_{p}\left(k r_{s 1}\right) e^{-i p \varphi_{s 1}}
\end{aligned}
$$

and the functions $T_{m n}\left(\theta_{a p}\right)$ are the combinations of the Legendre polynomials as given in [11]. Due to the Fredholm second kind nature of (16), its solving is remarkably stable and always convergent to the exact solution when the number of equations is increased. However, a special care should be taken for numerical summation of the Bessel function series such as in (9) and (10).

\section{RESUlts OF NUMERICAL OPTIMIZATION}

Based on the numerical solution of (16), we find the far-field radiation pattern as

$$
H^{\mathrm{tot}}(\theta)=\sum_{n=-\infty}^{\infty} i^{n} X_{n} e^{i n \theta}
$$

where $\theta=\pi-\varphi$

$$
\begin{aligned}
X_{n} & =\eta\left[J_{n}\left(k r_{s}\right) e^{-i n \varphi_{s}}+x_{n} J_{n}^{\prime}(k a)\right] s_{n} t_{n} / Q_{n} \\
s_{n} & =J_{n}^{\prime}(k c) H_{n}(k c)-J_{n}(k c) H_{n}^{\prime}(k c) \\
t_{n} & =J_{n}^{\prime}\left(k_{\epsilon} d\right) H_{n}\left(k_{\epsilon} d\right)-J_{n}\left(k_{\epsilon} d\right) H_{n}^{\prime}\left(k_{\epsilon} d\right)
\end{aligned}
$$

and $Q_{n}$ is defined in (11).

On integrating the relevant Poynting vector, the total radiation power $P^{\text {tot }}$ is derived as

$$
P^{\text {tot }}=\frac{2 Z_{0}}{k} \sum_{n=-\infty}^{\infty}\left|X_{n}\right|^{2}
$$

and the directivity of the antenna system is found as

$$
D^{\text {tot }}=\frac{2 Z_{0}}{k P^{\text {tot }}}\left|\sum_{n=-\infty}^{\infty} i^{n} X_{n}\right|^{2} .
$$

We used the derived equations for numerical optimization of the complete antenna system considering the total directivity as the aim function to be maximized. The efficiency of the algorithm can be understood from the fact that for a fixed geometry as given below, the complete solution of 50 equations guaranteed a uniform three-digit accuracy in far-field patterns and required $3 \mathrm{~s}$ with a SUNSPARC-20 workstation.

In Fig. 2, we present some sample far-field patterns of the cylindrical antenna with reflector parameters $k a=31.4$ and $\theta_{a p}=30^{\circ}(D=5 \lambda, f / D=0.5)$, feed parameters $k b=2.6$ and $\beta=0$ (the edge illumination from -9.2 to $-10 \mathrm{~dB}$ depending on $r_{0}$ ), and radome parameters $c=6 \lambda$ and $\epsilon_{r}=2$. A well-known effect is the focal shift of the feed: the latter should be placed nearer to the reflector than

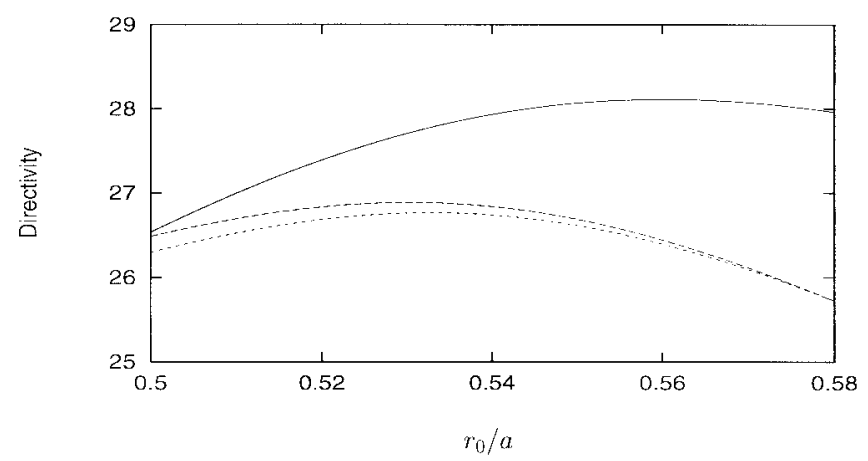

(a)

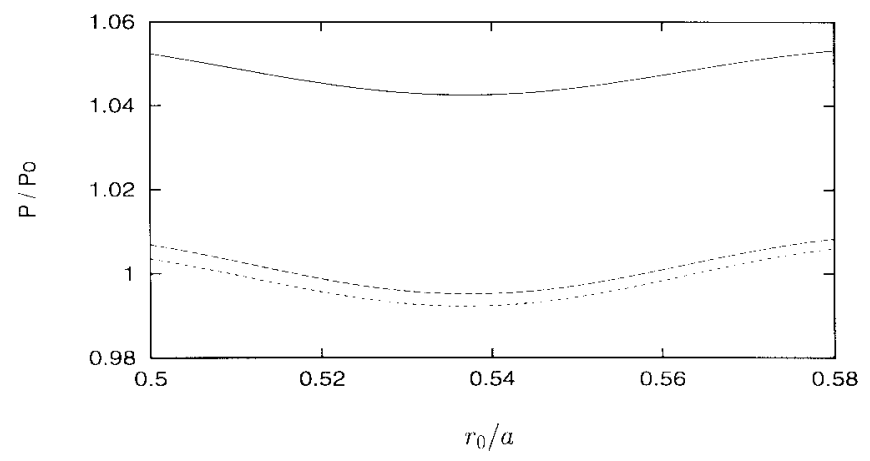

(b)

Fig. 3. (a) Directivity and (b) normalized total radiation power as functions of the feed position $r_{0}$ for the reflector in free space (dotted curve), in the radome of the "rule-of-thumb" parameters $t=0.5 \lambda_{\epsilon}$, $L=a \cos \left(\theta_{a p}\right)=4.330 \lambda, c=6 \lambda$ (dashed curve), and in the fully optimized radome/reflector system (solid curve).

the geometrical optics (GO) focus $r_{0}=a / 2$, to achieve a higher directivity [9]. In Fig. 3(a) we show several comparative plots of the directivity versus the feed position. They confirm this result of approximate high-frequency analysis, although in our numerical example the reflector is of moderate size. The similar dependences of the normalized total radiation power are given in Fig. 3(b). They reveal that the optimum positioning of the feed is accompanied with a slight reduction of the radiated power.

Another well-known recommendation, based on the analysis of the plane wave incident on a flat slab, is to take the radome thickness as $1 /\left(2 \epsilon_{r}^{1 / 2}\right)$ of the free-space wavelength $\left(t=0.5 \lambda_{\epsilon}\right)$. However, in reality neither the radome is flat nor the radiation field is a plane wave. The presence of a curved PEC reflector inside the radome is an additional reason for more complicated scattering. This is especially important for smaller radomes. Full-wave analysis by solving (7) shows that the actual optimum thickness yielding a maximum directivity is shifted to a smaller value [Fig. 4(a)]. Note that the normalized radiation power displays a similar oscillating behavior [Fig. 4(b)], with a unit value around the optimum thickness points.

If the thickness has been optimized, then varying the radome radius and the reflector position has a smaller effect on the directivity. However, the feed position should be corrected with respect to the free-space optimum after finding the best thickness (see Fig. 3.) Returning to Fig. 2, compare two free- 


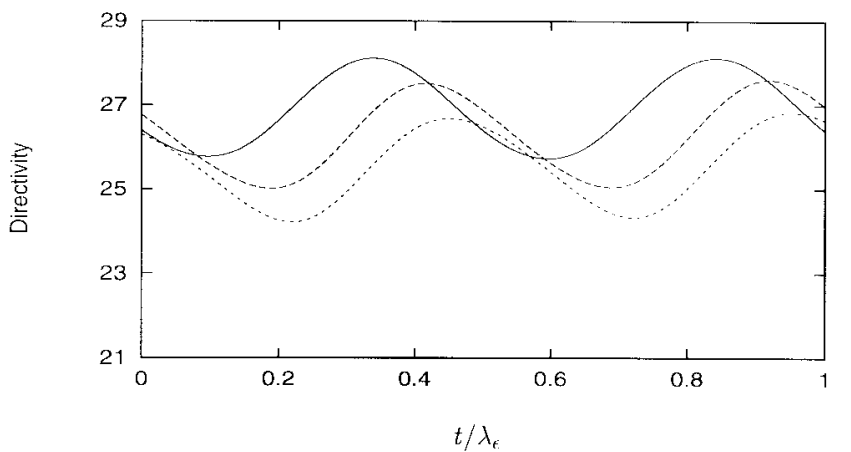

(a)

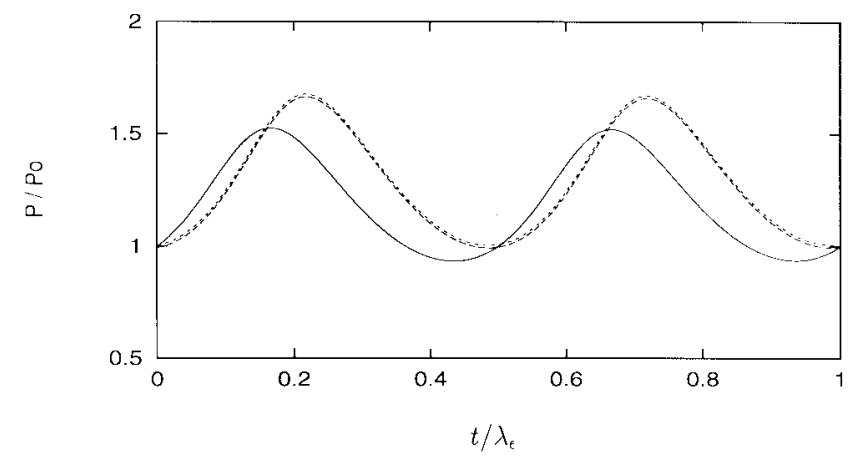

(b)

Fig. 4. (a) Directivity and (b) normalized total radiation power as functions of the radome thickness $t$ in the optimum system $\left(r_{0}=0.560 a\right.$, solid curve) and in the "rule-of-thumb" radome with the feed position $r_{0}=0.533 a$ (dashed curve) and $r_{0}=0.5 a$ (dotted curve).

space radiation patterns (for nonoptimized in-GO-focus feed, and for the optimized one) with a pattern for the completely optimized reflector-in-radome antenna system. One can see that the improvement of the directivity is obtained at the expense of the first sidelobe level. To have both the directivity and the sidelobe level reduced, one should search for an extremum of another aim function taking into account the two parameters simultaneously.

Figs. 5(a) and 6(a) give a vision of the dependence of the directivity on the radome inner radius and the radome position, respectively. The both dependences display an oscillating behavior around some average level determined mainly by the size of the reflector. In Figs. 5(b) and 6(b) the similar dependences of the normalized total radiation power are given. Like the thickness dependence, they show that in the optimized antenna system this value is near unity.

Notice that the scale of $c$ or $L$ variations providing the variations of directivity from its minimum to maximum value is $\lambda / 4$ while the scale of the relevant variations of the radome thickness is, respectively, $\epsilon_{r}^{1 / 2}$ times less. Dependence of the directivity on the feeder position is, however, of another scale determined by the reflector radius $a$ since it is controlled, basically, by the laws of geometrical optics.

It is also worth noting that the effect of the radome radius and the reflector location on directivity is considerably greater in the optimized system than in nonoptimized one. This is explained by the resonant character of the optimization, due to accurate taking into account all the multiple scattering

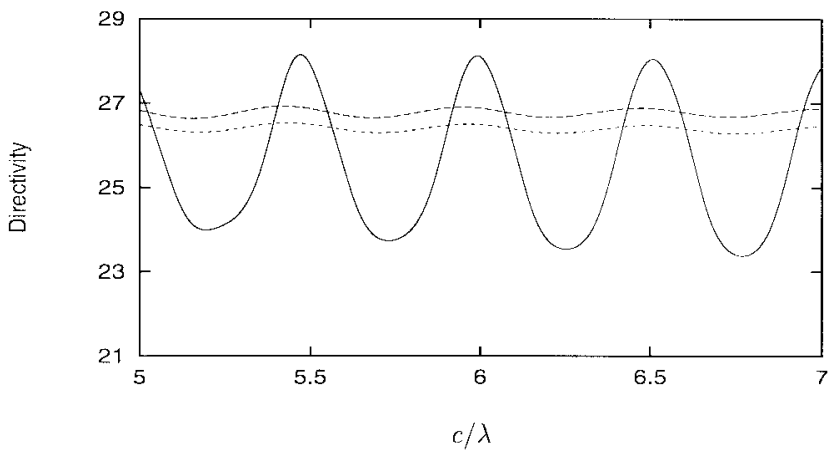

(a)

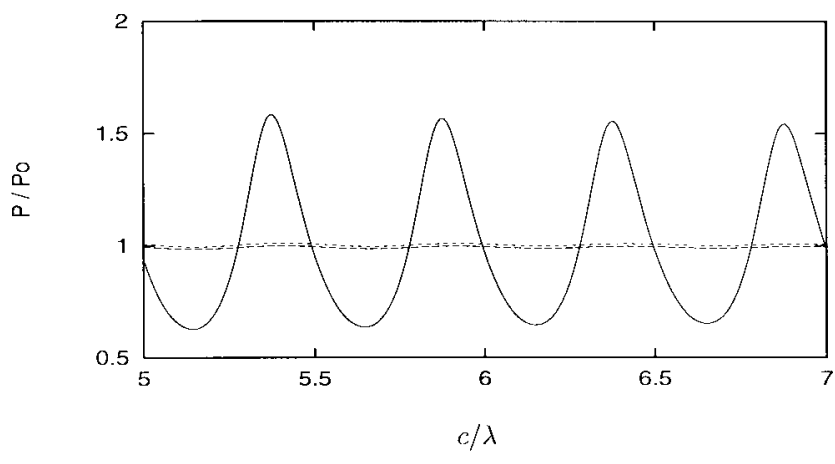

(b)

Fig. 5. (a) Directivity and (b) normalized total radiation power as functions of the radome inner radius $c$ (the legend is the same as in Fig. 4).

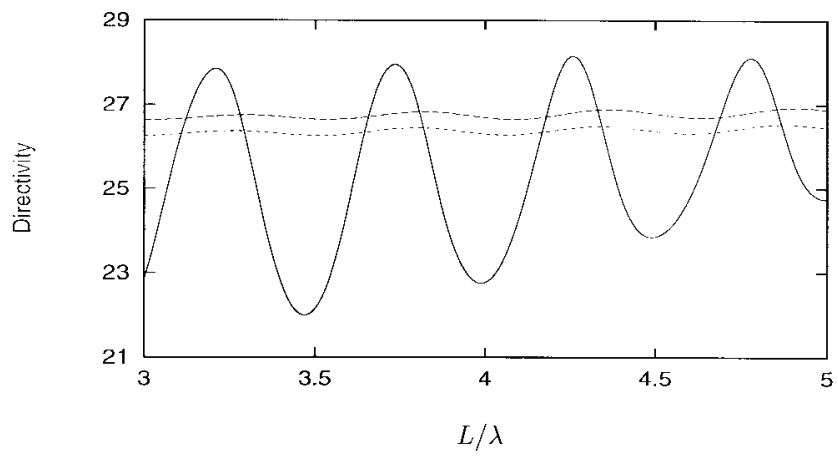

(a)

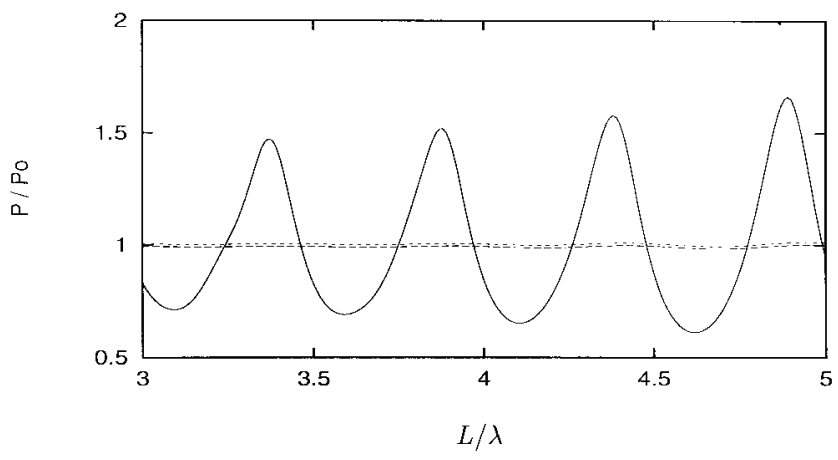

(b)

Fig. 6. (a) Directivity and (b) normalized total radiation power as functions of the radome position $L$ (the legend is the same as in Fig. 4).

interactions between the radome and the reflector. We may say that the radome acts as a resonant lens in this case, compressing the radiation along the main beam direction. 


\section{CONCLUSIONS}

One can see that taking a $\lambda /\left(2 \epsilon_{r}^{1 / 2}\right)$ radome thickness is actually a good recommendation even for a $5-\lambda$ reflector in a $6-\lambda$ radome. If it is satisfied, the effect of varying the radome position is minimum. However, provided that the thickness is slightly unmatched one can enhance the directivity. This is obviously the result of a greater sensitivity of the unmatched radome to the multiple internal reflections. It is only due to a resonance that the directivity is greater than of the same reflector in free-space.

Summarizing, we note that the multiple scattering in the reflector-in-radome antenna system is generally more significant for the optimum radome design than both nonplanewave effects and the curvature of the radome. Therefore, the IE analysis of the whole system is mandatory and can be hardly substituted by studying an approximately formulated problem. For the same reason, solving the full-wave IE with conventional MoM is also insufficient since the latter is heavily inaccurate near the resonances [16]. Contrary to this, the MoRbased numerical solution is uniformly accurate and can be efficiently used in the numerical antenna optimization code.

\section{REFERENCES}

[1] J. D. Kraus, Antennas. New York: McGraw-Hill, 1988.

[2] R. F. Harrington and J. R. Mautz, "An impedance sheet approximation for thin dielectric shells," IEEE Trans. Antennas Propagat., vol. AP-23, pp. 531-534, 1975

[3] D. C. F. Wu and R. C. Rudduck, "Plane wave spectrum-Surface integration technique for radome analysis," IEEE Trans. Antennas Propagat., vol. AP-22, pp. 497-500, 1974.

[4] P. D. Einziger and L. B. Felsen, "Rigorous asymptotic analysis of transmission through a curved dielectric slab," IEEE Trans. Antennas Propagat., vol. AP-31, pp. 863-869, 1983.

[5] X. J. Gao and L. B. Felsen, "Complex ray analysis of beam transmission through two-dimensional radomes," IEEE Trans. Antennas Propagat., vol. AP-33, pp. 963-975, 1985.

[6] L. B. Felsen, N. Subramaniam, and K. Arichandran, "Equivalence relation between partial angular harmonic and ray-type Green's functions for a cylindrical dielectric layer," IEEE Trans. Antennas Propagat., vol. 38, pp. 1273-1279, 1990.

[7] J.-H. Chang and K.-K. Chan, "Analysis of a two-dimensional radome of arbitrary curved surface," IEEE Trans. Antennas Propagat., vol. 38, pp. 1565-1568, 1990.

[8] R. K. Gordon and R. Mittra, "Finite element analysis of axisymmetric radomes," IEEE Trans. Antennas Propagat., vol. 41, pp. 975-980, 1993.

[9] H. Ling, S.-W. Lee, P. T. C. Lam, and W. V. T. Rusch, "Focal shifts in parabolic reflectors," IEEE Trans. Antennas Propagat., vol. AP-33, pp. 744-748, 1985.

[10] G. A. Suedan and E. V. Jull, "Beam diffraction by planar and parabolic reflectors," IEEE Trans. Antennas Propagat., vol. 39, pp. 521-527, 1991.

[11] T. Oǧuzer, A. Altintaş, and A. I. Nosich, "Accurate simulation of reflector antennas by the complex source-Dual series approach," IEEE Trans. Antennas Propagat., vol. AP-43, pp. 793-801, 1995.

[12] H. Cory and P. J. B. Clarricoats, "Reflection coefficient at the feed of a radome-covered reflector antenna," Proc. Inst. Elect. Eng., vol. 129, pt. H, no. 4, pp. 145-152, 1982.

[13] P. J. B. Clarricoats, C. G. Parini, and M. S. A. S. Rizk, "Performance of radome-covered reflector antennas," Proc. Inst. Elect. Eng., vol. 129, pt. H, no. 4, pp. 153-160, 1982.

[14] Microwave Antenna Theory and Design, S. Silver, Ed. London: Peter Peregrinus Ltd, 1984.

[15] S. S. Vinogradov, E. D. Vinogradova, A. I. Nosich, and A. Altintaş, "Analytical regularization based analysis of a spherical reflector symmetrically fed by a scalar beam," SIAM J. Appl. Math., 1998, submitted for publication.

[16] G. L. Hower, R. G. Olsen, J. D. Earls, and J. B. Schneider, "Inaccuracies in numerical calculations of scattering near natural frequencies of penetrable objects," IEEE Trans. Antennas Propagat., vol. 41, pp. 982-986, 1993.

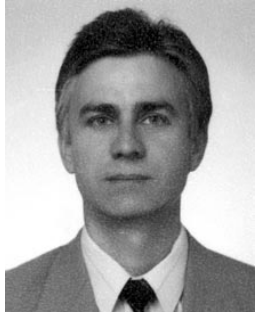

Vladimir B. Yurchenko was born in Kharkov, Ukraine, in 1957. He received the M.S. degree (with honors) from the Physics and Technology Department, Kharkov Polytechnic Institute, Kharkov, Ukraine, the Ph.D. degree in solid-state physics from the Kharkov Polytechnic Institute, and the D.Sc. degree from the Institute of Radiophysics and Electronics (IRE), Ukrainian Academy of Sciences, Kharkov, in 1980, 1984, and 1994, respectively.

From 1984 to 1995, he was with Department of Materials for Electronics and Solar Cells, Kharkov Polytechnic Institute, Ukraine, as a Research Scientist and an Associate Professor. His research interests there included the theory of hot-electron transport, photovoltaics, and electrical instability in semiconductors. Since 1995, he has been with the IRE as a Senior Research Scientist in the Department of Nonlinear Dynamics of Electronic Systems. Since 1996, he has been a member of the visiting faculty at the Department of Electrical and Electronics Engineering, Bilkent University, Ankara, Turkey. His current interests include computer simulation of electron transport in nanostructures, dynamical chaos in electronic devices, and wave scattering in electromagnetic systems.

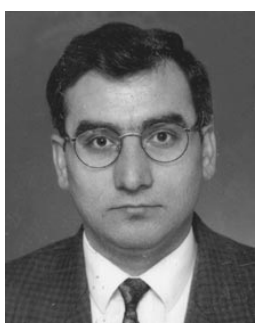

Ayhan Altintaş (''82-M'86-SM'93) was born in Yozgat, Turkey, on March 29, 1958. He received the B.S. and M.S. degrees from the Middle East Technical University (METU), Ankara, Turkey, in 1979 and 1981, respectively, and the Ph.D. degree from The Ohio State University, Columbus, $\mathrm{OH}$, in 1986, all in electrical engineering.

From 1981 to 1987, he was with the ElectroScience Laboratory of The Ohio State University At present, he is a Professor at the Department of Electrical and Electronics Engineering, Bilkent University, Ankara, Turkey. He has held research fellowship and Guest Professor positions at Australian National University, Canberra, Australia, Tokio Institute of Technology, Tokio, Japan, and Technical University of Munich, Munich, Germany. His research interests include electromagnetic radiation and scattering, microwaves, fiber optics, and integrated optics.

Dr. Altintaş received the ElectroScience Laboratory Outstanding Dissertation Award of 1986, the IEEE 1991 Outstanding Student Branch Counselor Award, the 1991 Research Award of the Professor Mustafa N. Parlar Foundation of METU, and the 1996 Young Scientist Award of TUBITAK. He is a member of Sigma Xi and Phi Kappa Phi.

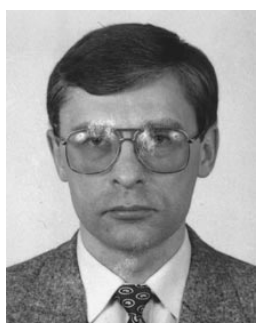

Alexander I. Nosich (M'94-SM'95) was born in 1953 in Kharkov, Ukraine. He received the bachelor's degree from the Radio Physics Department of Kharkov State University in 1975 . He received the Ph.D. and D.Sc. degrees in radio physics from the same university in 1979 and 1990, respectively.

Since 1978, he has been on research staff of the Institute of Radiophysics and Electronics (IRE) of the Ukrainian Academy of Sciences, in Kharkov. In 1992 to 1998 he held research fellowships and visiting professorships in Bilkent University, Ankara, Turkey; Kumamoto University, Gifu University and Chuo University, Tokyo, Japan; University of Rennes 1, France; and Torino Polytechnical University, Italy. Currently he is with IRE, Kharkov, as a leading scientist in the Department of Computational Electromagnetics. His research interests include analytical pregularizations, free-space and open-waveguide scattering, complex mode behavior, radar cross-section analysis, and antenna simulation.

Dr. Nosich was the organizer and chairman of the IEEE AP-S East Ukraine Chapter in 1995, the first one in the Former Soviet Union. Since 1995, he has been on the editorial board of Microwave and Optical Technology Letters. From 1990 to 1998, he was the organizer and TPC Cochairman of a series of international conferences on Mathematical Methods in Electromagnetic Theory (MMET) held in the Ukraine. 